\title{
Fracture Investigation of Hollow Cylindrical Tether during Space Debris Impact
}

\author{
By Kazumasa SASAHARA, ${ }^{1)}$ Yoshiyuki Uwamino, ${ }^{1)}$ \\ Sunao HASEGAWA, ${ }^{2)}$ and Kanjuro MAKIHARA ${ }^{1)}$ \\ ${ }^{1)}$ Department of Aerospace Engineering, Tohoku University, Sendai, Japan \\ ${ }^{2)}$ Institute of Space and Astronautical Science, Japan Aerospace Exploration Agency, Sagamihara, Japan
}

(Received June 24th, 2017)

\begin{abstract}
Space debris poses a serious threat and the problem must be solved. Space debris should be removed to maintain the space environment. One of the techniques to remove it is an electrodynamic tether system (EDTS). To improve the toughness of the tether used in EDTS, this study investigated the tether shape of a hollow cylinder instead of the conventional solid cylinder. When the mass and length of a hollow tether are the same as those of the solid tether, the hollow cylindrical tether has a larger diameter. Thus, the toughness against small debris collisions will be improved. To investigate the damage to the hollow tether following a debris collision, hypervelocity impact experiments were performed using a two-stage light-gas gun. Through the impact experiments, the relationship between the damage to the hollow tether and the impact condition of the debris was revealed. It was confirmed that when the impact angle increased the damage area increased as well. The experiments validated that a hollow tether is superior to a solid tether in terms of resistance to debris collisions. In addition, the importance of considering the influence of the impact angle was shown by an evaluation of the lifetime of the hollow tether.
\end{abstract}

Key Words: Hollow Cylindrical Tether, Debris Cloud, Oblique Impact, Residual Ratio, Survival Probability

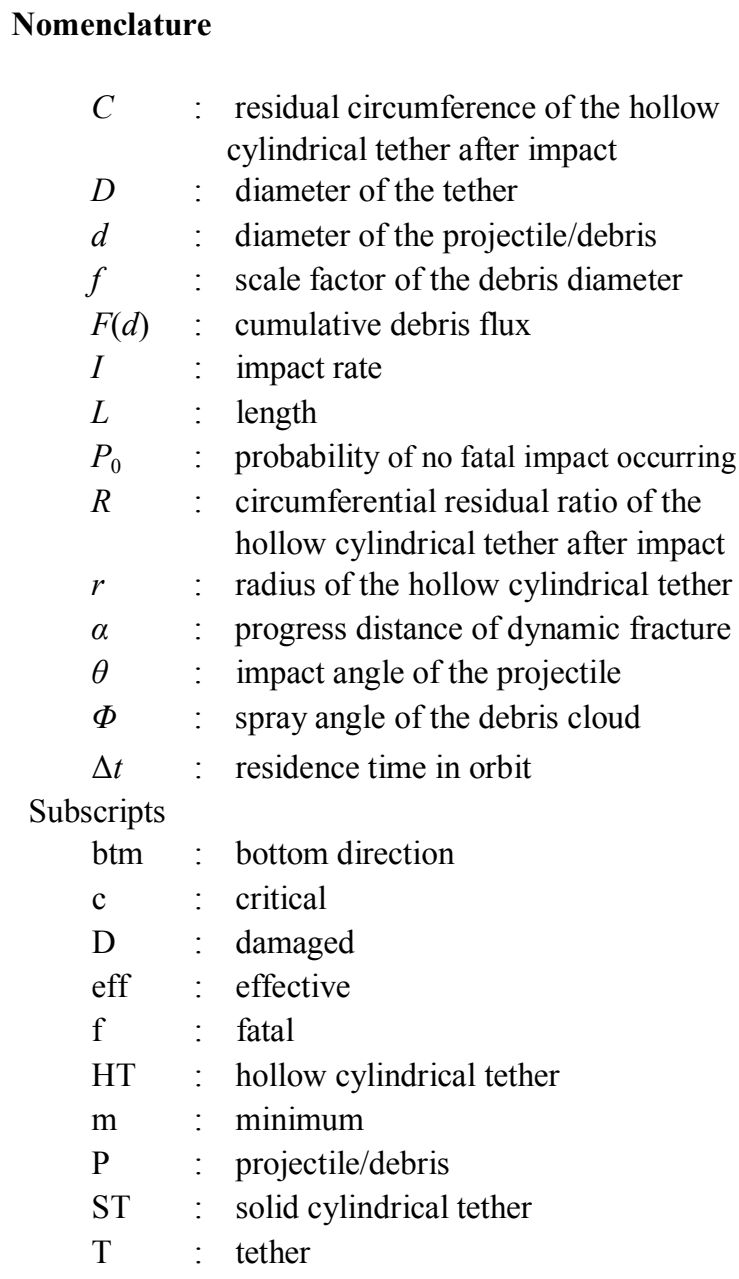

$$
\begin{array}{ll}
\text { top } & : \text { top direction } \\
X Y & : \quad X Y \text {-plane value } \\
Z X & : \quad Z X \text {-plane value }
\end{array}
$$

\section{Introduction}

Recently, the amount of space debris has been on the rise. This could lead to a serious threat to further space development. Space debris consists of nonfunctional spacecraft, the final stage of rockets, and fragments from satellite breakups. Satellites under operation can be damaged by space debris collisions. This could be a matter of life and death for the individuals boarding them. Currently, there are approximately 500,000 pieces of space debris with their sizes varying from $1 \mathrm{~cm}$ to $10 \mathrm{~cm}$. Moreover, there are approximately $100,000,000$ pieces of space debris called small debris that cannot be tracked with any observation devise from the ground, and therefore, cannot be avoided in space. Even small debris with a size less than $1 \mathrm{~cm}$ has a very large energy as they travel at very high velocities in Earth orbit. One scenario that generates new small debris is a collision between large debris. The amount of debris will continue to increase by self-reproduction if debris is not removed. In addition, this implies that the probability of a collision between a satellite and debris will increase. Therefore, the removal of debris is an urgent issue for future space development. A recent method of space debris removal from orbit around the Earth is an electrodynamic tether (EDT) system, depicted in Fig. 1. The EDT system consists of a conducting tether and an emitter. The EDT system can remove space debris as follows. First, a conducting tether is attached mechanically to the removal-target satellite. The emitter starts to emit electrons 


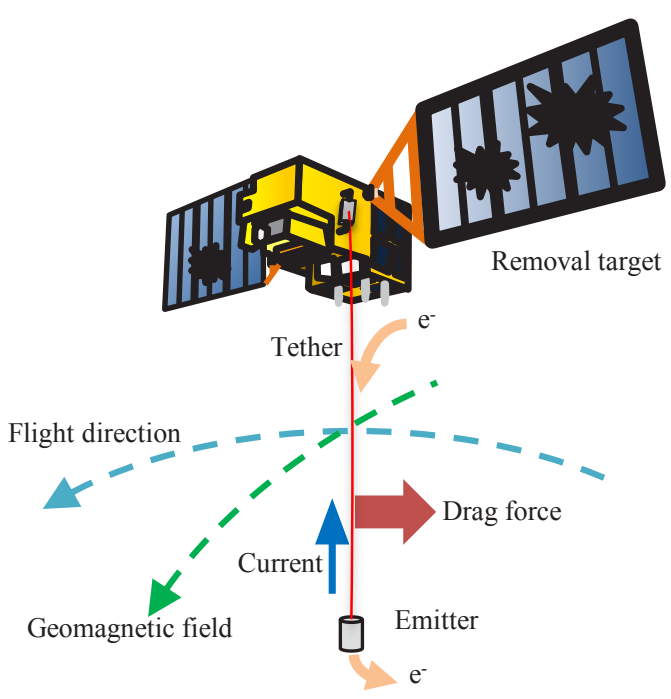

Fig. 1. Schematic of electrodynamic tether system.

with surrounding plasma. A pseudo circuit can be created with the conducting tether and the space atmosphere by collecting electrons from plasma in the space environment and by emitting electrons at the end. Because of the motion of the electrons, electric current flows in the longitudinal direction of the conducting tether. Lorentz force is induced by the interference between the current and the geomagnetic field. The removal-target satellite is decelerated by Lorentz force as this force acts in the direction opposite to the flight direction of the target. The slower velocity of the satellite breaks the equilibrium between centrifugal and gravitational forces. Finally, the satellite deviates from its orbit and falls to Earth, where it burns up in the atmosphere of the Earth. The EDT system is relatively low cost because it does not require any propellant to de-orbit the satellite. However, the shape of the conducting tether is an extremely long configuration. Therefore, the collision rate of debris that has a sufficiently large size to sever the conducting tether is higher. This is because the conducting tether can be easily severed by collision with small debris; consequently, any severed tether forms new debris. Therefore, the construction of a tether that is difficult to sever is required.

Studies on the collision between a tether and debris have been carried out all over the world, and some achievements have been reported. Hirayama et al. ${ }^{1)}$ proposed a method for calculating the survival probability, which is one of the indexes that evaluates the survival performance, for a single solid cylindrical tether and the double solid cylindrical tether. Anselmo and Pardini2) and Anz-Meador ${ }^{3)}$ proposed a calculation method for the survival probability. These proposed methods were compared by Pardini et al. ${ }^{4)}$ This comparison showed that the double tether has a greater survival probability than single tether.

Various shapes for the conducting tether were proposed and studied to improve the survivability. However, the success rate of the debris removal mission did not have a satisfactory value when these proposed tethers were used. ${ }^{5)}$ Survival probability depends on the debris-flux model and the construction of the conducting tether. Pardini et al. ${ }^{4)}$ showed that all of the methods, shown in Refs. 1-3), come to the
Table 1. Relationship between diameter and lifetime in orbit.

\begin{tabular}{ccccc}
\hline Diameter $D$ & $D<1 \mathrm{~mm}$ & $D=2.5 \mathrm{~mm}$ & $D>5 \mathrm{~mm}$ & $D>10 \mathrm{~mm} \quad D>25 \mathrm{~mm}$ \\
\hline Operation time & 10 days & $40-50$ days & 1 year & several years $>10$ years \\
\hline
\end{tabular}

common conclusion that the lifetime of a single solid cylindrical tether depends primarily on its diameter, as shown in Table 1. According to Ref. 4), the single tether with a diameter smaller than $1 \mathrm{~mm}$ may survive intact for less than 10 days for all orbits and tether lengths. When the diameter is $2.5 \mathrm{~mm}$ then the average lifetime is about 40-50 days for a $5-\mathrm{km}$ tether and $800-\mathrm{km}$ altitude. When the diameter is larger than $5 \mathrm{~mm}$ then the single tether may survive intact for less than 1 year for all orbits and tether lengths. When the diameter is greater than $10 \mathrm{~mm}$ then debris collision may no longer be a threat for missions. The diameter is greater than $25 \mathrm{~mm}$ then the single tether may act as a tether for a long time, ranging from a few decades to more than a century. It is common knowledge that the lifetime depends on both tether length and orbital configuration, whereby increasing the tether length results in a decrease in lifetime. Consequently, as the diameter of a tether is increased, the lifetime that the tether will operated as an EDT system tends to increase. However, it is difficult to increase the diameter due to the mass problem. Therefore, it is preferred solution to have a large diameter for the conducting tether, to improve performance, while not increasing the mass. Recently, a hollow cylindrical tether has been proposed ${ }^{6)}$ as a preferable shape for the tether. However, present research on hollow cylindrical tethers does not provide the necessary understanding of the damage caused by debris collision.

\section{Objectives of This Study}

This study had two objectives. The first was the evaluation of the survivability of a hollow cylindrical tether based on the hypervelocity horizontal impact experiment. Here, a horizontal collision means a perpendicular collision with the longitudinal axis of the tether. In Ref. 6), impact experiments were carried out and these results were discussed without considering the impact angles. In this study, the impact condition was described by using the impact angle in the horizontal plane to focus on its influence. This was done so that the difference between the hollow and solid cylindrical tethers could be evaluated. The diameter of a hollow cylindrical tether is larger than that of a solid cylindrical tether when their masses and lengths are the same values. ${ }^{6)}$ According to Ref. 6), when the hollow cylindrical tether collides with small debris that has an enough size to sever the solid cylindrical tether, it is not severed. The hollow cylindrical tether is expected to have a greater survivability against collisions between small debris than the solid cylindrical tether. To investigate the fracture of a hollow cylindrical tether against horizontal debris collisions, a hypervelocity horizontal impact experiment was carried out. The two-stage light-gas gun owned by the Institute of Space and Astronautical Science (ISAS) of Japan Aerospace Exploration Agency (JAXA) was used. By utilizing the observations and results of this experiment protective performance, which is one of the indexes to assess survivability, of a hollow cylindrical tether was evaluated.

The second objective was the evaluation of the influence of debris impact angle based on the hypervelocity oblique impact experiment and estimation of the survivability of a hollow 
cylindrical tether. Here, an oblique impact means an oblique collision with the longitudinal axis of the tether. When debris collides with a hollow cylindrical tether at an oblique angle, fragments are generated inside the hollow cylindrical tether and they are considered to spread wider than the perpendicular collision. The hypervelocity oblique impact experiment was conducted to investigate the fracture of a hollow cylindrical tether against an oblique debris collision. Comparison of the fractures due to horizontal or oblique debris collisions reveals a relationship between the fracture of a hollow cylindrical tether and the collision angle of debris. In addition, the protective performance was evaluated to show the influence of collision angle on the success rate of debris removal mission.

This paper constitutes of the following sections. Section 3 summarizes the space-debris horizontal impact experiment, considerations, and results. In addition, the relationship between the debris-impact points on a hollow cylindrical tether and the damage to the tether is considered. At the end of section 3, a reason for the variation in damage is considered. Section 4 describes the use of the proposition to calculate the survivability of a tether using impact angle. Section 5 compares the survivability of the proposed hollow cylindrical tether with that of a conventional solid cylindrical tether to evaluate the protective performance of the former under a debris impact. Section 6 summarizes the space-debris oblique impact experiment, considerations, and results. In addition, the relationship between the debris-impact angle on a hollow cylindrical tether and the damaged region of the tether is considered. Section 7 compares the survivability of a hollow cylindrical tether under the conditions that the impact angle was or was not considered. Finally, section 8 concludes this paper.

\section{Horizontal Impact Experiments}

\subsection{Two-stage light-gas gun}

A two-stage light-gas gun owned by ISAS/JAXA was used to carry out the horizontal impact experiment. The two-stage light-gas gun is shown in Fig. 2. A spherical polycarbonate projectile with a diameter of $7.14 \mathrm{~mm}$ was used to simulate space debris. A melting point of polycarbonate is lower that of than aluminum. A polycarbonate projectile is considered to melt more than an aluminum projectile when it collides with a tether. This means a polycarbonate projectile spreads and collides with a wider range compared with an aluminum one. Therefore, the damage on a hollow cylindrical tether becomes greater. From a viewpoint of safety factor, using a polycarbonate projectile brings a strict evaluation of the damage caused by debris collision. The hollow cylindrical tether described in the next subsection was used as a target

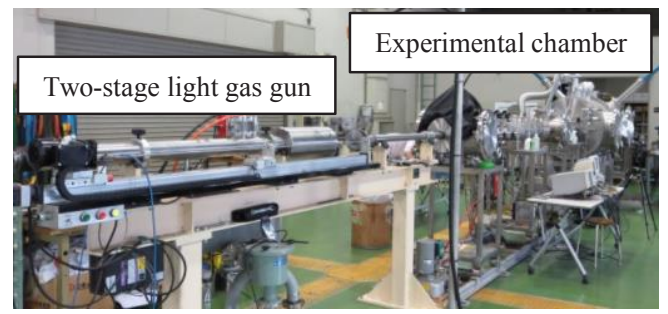

Fig. 2. Two-stage light-gas gun at ISAS/JAXA. and placed inside of an experimental chamber. The impact velocity was set to $7 \mathrm{~km} / \mathrm{s}$. A high-speed camera (Hyper Vision HPV-X, Shimadzu Corporation) was used to photograph the impact event.

\subsection{Hollow cylindrical tether at impact experiments}

The hollow cylindrical tether used as a target for the experiments and the associated equipment are shown in Fig. 3. The surface of the hollow cylindrical tether that was expected to be impacted by the projectile and a view of its lateral direction are depicted in Figs. 3(a) and 3(b), respectively. The black cylindrical objects that are attached to both ends of the tether were the jigs used to maintain the tether shape of a hollow cylinder. The hollow cylindrical tether had a diameter of $146 \mathrm{~mm}$, and was formed from stranded 0.12-mm-diameter tin-plated copper wires. They were knitted and the seam was not adhered. They were spaced at about $1.34 \mathrm{~mm}$ in the circumferential direction and $1 \mathrm{~mm}$ in the axial direction. The setup of the hypervelocity experiment of the hollow cylindrical tether and the projectile is shown in Fig. 4. Figure 4(a) shows the setup of the hypervelocity experiment along with the cross section of the hollow cylindrical tether and the projectile within the field of the view of the high-speed camera. Figure 4(b) shows the setup of the hypervelocity impact experiment within the field of the view from the horizontal direction to the $Y$-axis. In $X Y$-plane, the $X Y$-plane impact angle $\theta_{X Y}$ is defined as the angle that is formed between the standard plane passed through the center of the hollow cylindrical tether and the projectile-flying line. In $Z X$-plane, the $Z X$-plane impact angle $\theta_{Z X}$ is defined as the angle that is formed between the cross section of the hollow cylindrical tether and the projectile-flying line. Here, the horizontal impact experiment was carried out under the condition of $\theta_{Z X}=0^{\circ}$. At each experimental condition, the projectile impacted on the same point of the surface of the hollow cylindrical tether, which is intersected with the

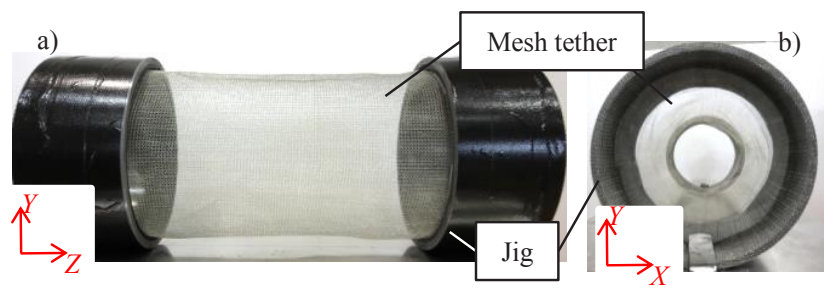

Fig. 3. Apparatus for impact experiment: a) impact surface side view and b) lateral view.

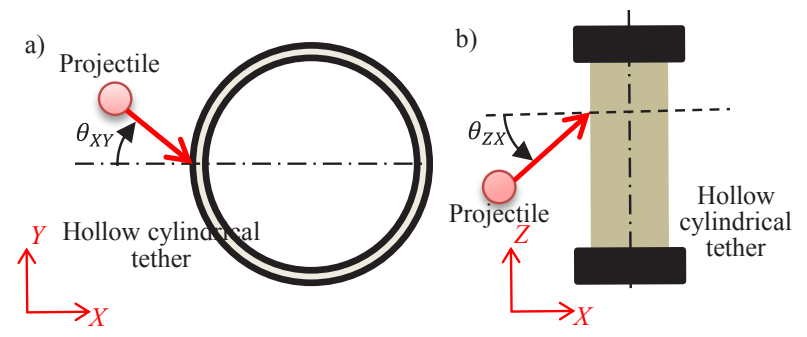

Fig. 4. Schematics of impact experiment performed on hollow cylindrical tether: a) $X Y$-plane view and b) $Z X$-plane view. 
horizontal plane. The relationship between the impact angle of the projectile and the damage to the hollow cylindrical tether is described in the next subsection.

\subsection{Results of horizontal impact experiments}

Figure 5 shows photographs of the hollow cylindrical tether after the horizontal impact experiments. Figures 5(a) and 5(b) were performed under the conditions of $\theta_{X Y}=3.4^{\circ}$ and $\theta_{X Y}$ $=45.2^{\circ}$, respectively. The impact direction of the projectile was directed out of the sheet. The small circle of damage at the rear side of the hollow cylindrical tether, in both figures, was the 1st destruction due to the impact of the projectile. The large amount of damage at the front end of the tether, in both figures, was the 2nd destruction due to the impact of the debris cloud. The debris cloud, which was generated by the impact on the front surface, caused significant damage to the rear surface of the tether. Therefore, the investigation hereafter focused on how the debris cloud spreads.

A photograph taken by a high-speed camera during the experiment is shown in Fig. 6. The presence of a debris cloud $6 \mu$ s after impact was observed. It was assumed that the front half of the debris cloud is spherical. To investigate the spread range of the debris cloud, three measurement points were set: the top edge, leading edge, and bottom edge.

Circular, triangular, and quadrate points in Fig. 7 represent the data collected at 2-intervals at the above three-kind measurement of the points for the impact that occurred in $\theta_{X Y}$ $=3.4^{\circ}{ }^{6)}$ The point of the projectile impact was used as the origin. In addition, the damaged part is indicated by arrows in the figure. An angle formed by the end points of the two arrows and the origin was defined as the damaged angle $\Phi_{\mathrm{D}}$. The experimental result showed that the damaged angle was smaller than the spray angle of the debris cloud. This fact revealed that a part of the debris cloud did not necessarily destroy the rear surface of the tether.

The three different types of measurement points, in Fig. 7, were linearly approximated with the least-squares method. The angles formed by the line $Y=0$ and the approximation line for the point on the top edge and bottom edge in the figure are denoted by $\Phi_{\text {top }}$ and $\Phi_{\text {btm }}$, respectively. The spray angle $\Phi_{\text {top }}+\Phi_{\text {btm }}$ indicates that the debris cloud spreads as a whole from the impact point. Figure 8 shows the relationship between $\Phi_{\mathrm{D}} /\left(\Phi_{\mathrm{top}}+\Phi_{\mathrm{btm}}\right)$ and $\theta_{X Y} \cdot{ }^{6)}$ According to Ref. 7), the influence of the impact velocity of the projectile can be disregarded because of the very small variance (the average impact velocity is $7.13 \mathrm{~km} / \mathrm{s}$ and the variance is 0.00160 ). The ratio of the damaged angle to the spray angle increased with the $X Y$-plane impact angle. This indicates that, as the $X Y$-plane

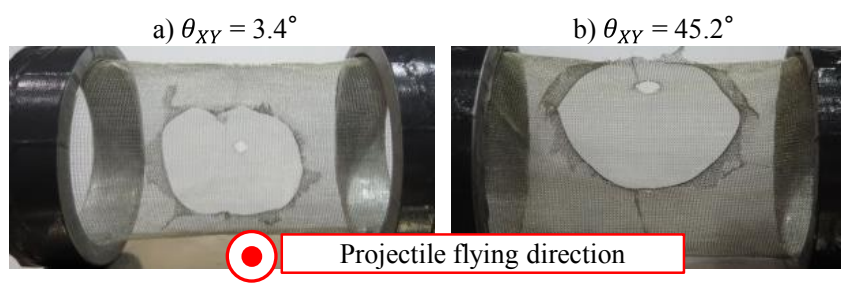

Fig. 5. Damage to tether after impact: a) $\theta_{X Y}=3.4^{\circ}$ and b) $\theta_{X Y}=$ $45.2^{\circ}$. impact angle increased, the debris cloud tended to be formed near the end of the circle that is inside of the cross section of the hollow cylindrical tether. When the debris cloud was formed in the upper portion of the hollow cylinder, it traveled a shorter distance before it impacted the rear of the tether. The impact with the rear of the tether thus occurred before the debris cloud significantly dispersed. This means that a portion of the debris cloud collided with the rear side of the tether

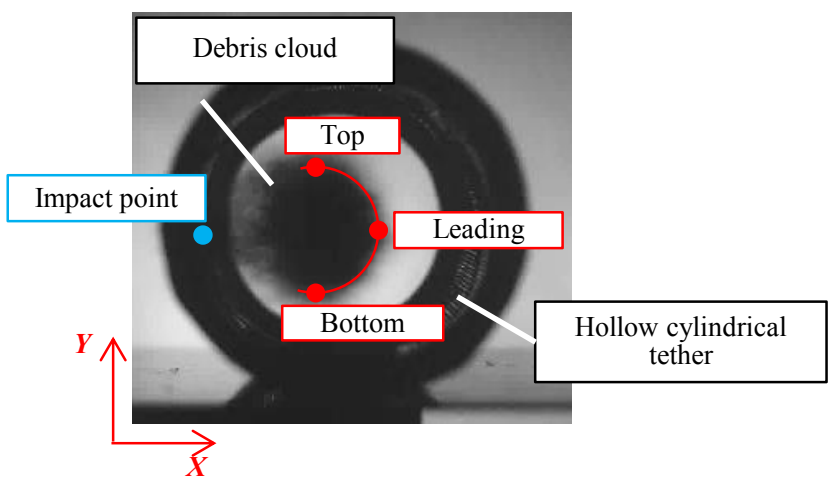

Fig. 6. Debris cloud generated inside of hollow cylindrical tether and analysis parameters ( $6 \mu \mathrm{s}$ after impact).

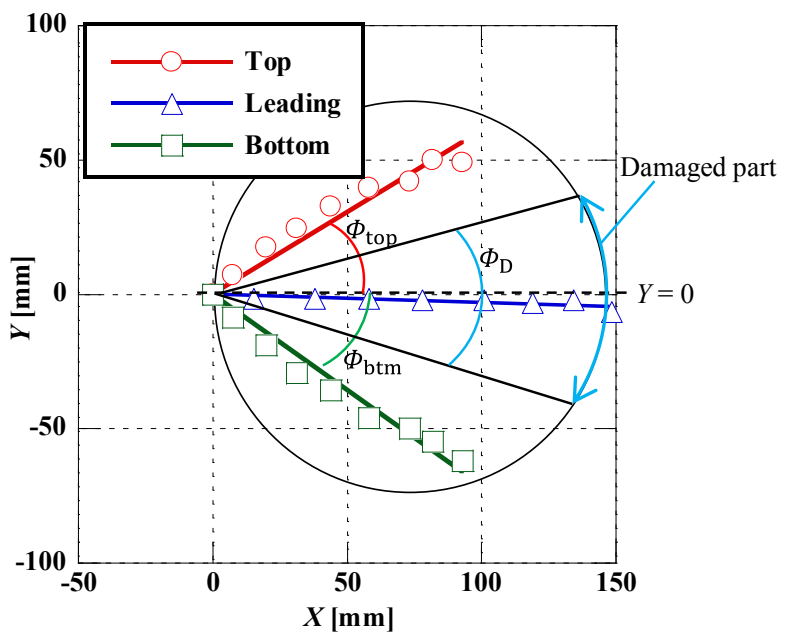

Fig. 7. Three measurement points, spray angle and damaged angle $\left(\theta_{X Y}=3.4^{\circ}\right)$.

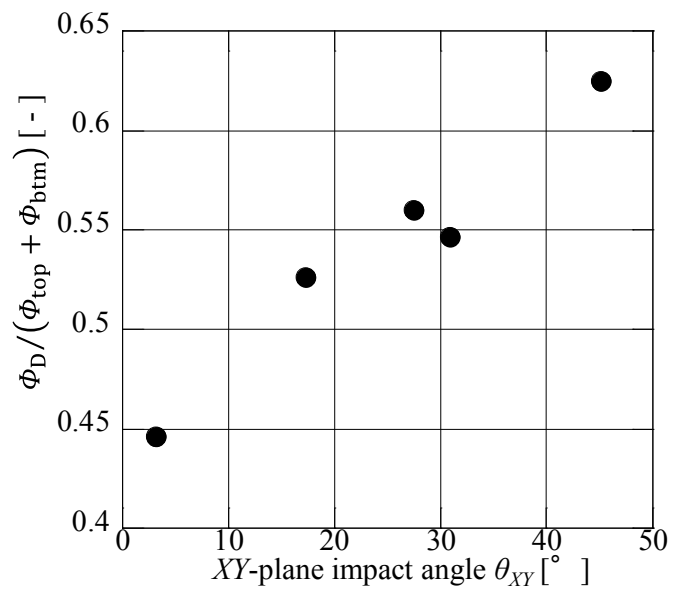

Fig. 8. Ratio of damaged angle to spray angle vs $X Y$-plane impact angle. 
while still containing relatively dense fragments. The dense fragments in the cloud possessed a greater capacity for creating damage. Accordingly, it was found that the ratio of the damage angle to the spray angle of the debris cloud increases with the $X Y$-plane impact angle.

\subsection{Residual length by horizontal impact}

The residual circumferential length $C$ is calculated by subtracting the sum of the circumferential length of the 1st and 2 nd destructions from the original circumferential length of the hollow cylindrical tether $2 \pi r$. The residual circumferential length $C$ is calculated by $C=2 \pi r-L_{\mathrm{D}}$, where the circumferential damaged length is the circumferential length as shown in Fig. 9. The circumferential damaged length $L_{\mathrm{D}}$ was measured between the circumferential end points of the damage to the projection on the $X Y$-plane. The ratio of the residual circumferential length $C$ against the original circumferential length of the hollow cylindrical tether $2 \pi r$ is the residual ratio $R \equiv C / 2 \pi r$.

Figure 10 shows the relationship between the residual ratio $R$ and the $X Y$-plane impact angle $\theta_{X Y}{ }^{6}{ }^{6}$ As the $X Y$-plane impact angle increases, the residual ratio tends to decrease. In other words, as the distance between the 1st and 2nd destructions becomes shorter, as described above, the damage increases because the debris cloud has a higher density. To investigate the influence of the projectile material, a spherical aluminum projectile with a diameter of $3.2 \mathrm{~mm}$ was used to

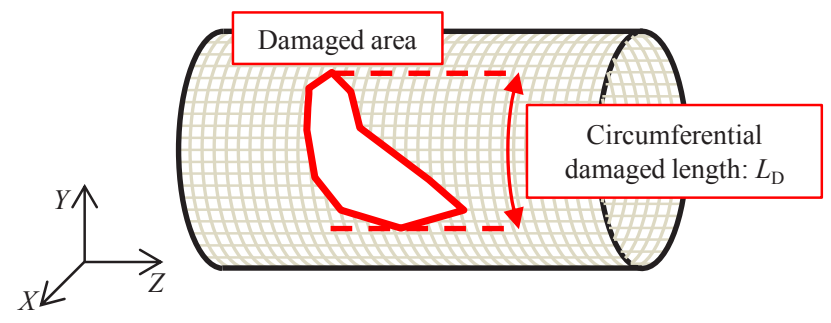

Fig. 9. Determination of damaged length $L_{\mathrm{D}}$.

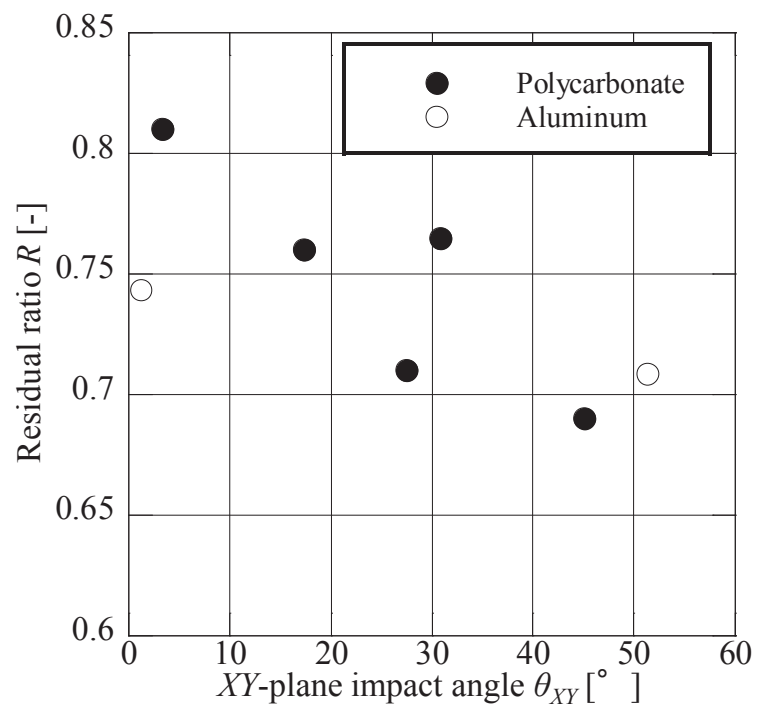

Fig. 10. Residual ratio $R$ vs $X Y$-plane impact angle $\theta_{X Y}$ in horizontal impact. simulate space debris. The two-stage light-gas gun cannot fire an aluminum projectile with a diameter larger than $3.2 \mathrm{~mm}$ because an aluminum projectile requires sabots to protect the equipment. Both projectiles (polycarbonate and aluminum) showed similar values for the residual ratio, as indicated in Fig. 10. This result showed that the material of the projectile has little influence on the residual ratio and that the impact velocity is the main factor governing the impact damage.

\section{Determination of Effective Cross-Sectional Area}

\subsection{Tether survival probability}

If the tether of the electrodynamic tether system is severed by small-debris during a mission to remove large satellites from Earth orbit, the mission would fail. In addition, the tether itself could become debris. Thus the survival performance of the tether in orbit must be evaluated. However, there is no evaluation method of the hollow cylindrical tether; therefore, a new method to evaluate the oblique impact of the hollow cylindrical tether is essential. Survivability is generally used as an index for evaluating survival performance. ${ }^{4,5}$

\subsection{Effective diameter for conventional tether}

The moment at which the projectile collided with the tether was considered. Figure 11 shows the orthogonal projection of the tether, which is parallel to the longitudinal axis. The effective diameter $D_{\text {eff }}$ of a tether is the width of the area where, if the center of a piece of debris passes through it, the tether would be severed. ${ }^{4,5)}$ In this subsection, the conventional concept for the effective diameter of a conventional solid cylindrical tether is discussed. The conventional solid cylindrical tether with the diameter $D_{\mathrm{ST}}$ would be severed if the one end of debris passed within $f_{\mathrm{c}} D_{\mathrm{ST}}$ of the tether center of axial symmetry. $f_{\mathrm{c}}$ is the critical scale factor, and its value varies among researchers. It is often set to about $2 / 3$ or $0.7 .^{5,8)}$ In addition, the melting of the tether is one of the factors that expands the damage area on the tether. ${ }^{9)}$ This expansion of the damaged length is called a progress distance of dynamic fracture and represented by $\alpha$ in this paper. When the diameter of debris is $d, D_{\text {eff }}$ can be calculated by $D_{\text {eff }}=f_{\mathrm{c}} D_{\mathrm{ST}}+d+2 \alpha D_{\mathrm{ST}}$ as illustrated

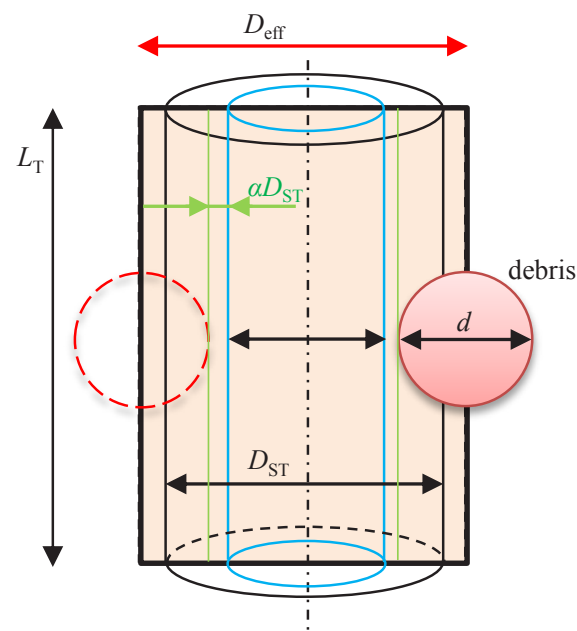

Fig. 11. Effective cross-sectional area of conventional tether (solid cylindrical tether). 
in Fig. 11

\subsection{Effective cross-sectional area for hollow tether}

An effective cross-sectional area for a hollow cylindrical tether was considered. The methods applied to conventional solid cylindrical tethers are not applicable. As shown in Ref. $6)$, the effective cross-sectional area for a hollow cylindrical tether is shown in Fig. 12. In this figure, $\theta_{X Y \mathrm{c}}$ is the critical $X Y$-plane angle when the residual ratio $R$ is equal to a critical residual ratio $R_{\mathrm{c}}$, which is the border value determining whether the hollow cylindrical tether is severed by collision with debris of diameter $d$. The effective cross-sectional area $D_{\text {eff }}$ of the hollow cylindrical tether is the width for which debris cannot sever the hollow cylindrical tether unless the center of debris does not pass this area. $D_{\text {eff }}$ depends on the diameter and impact angle of debris and three cases must be considered. Here, $d_{\mathrm{m}}$ is a critical debris diameter of the minimum size that is able to sever the hollow cylindrical tether; this was determined by the results of the hypervelocity impact experiments and numerical simulations. ${ }^{6}$ ) In case 1 , when debris with a diameter smaller than $d_{\mathrm{m}}$ collided with the hollow cylindrical tether, the tether was not severed by debris collision at any $X Y$-plane impact angle $\theta_{X Y}$. In case 2 , when debris with a diameter greater than or equal to $d_{\mathrm{m}}$ collided with the hollow cylindrical tether at a specific $X Y$-plane impact angle $\theta_{X Y}$ it caused enough damage for the tether to be severed. As mentioned in the preceding section, the damage to the hollow cylindrical tether caused by the debris collision increased with increasing $\theta_{X Y}$. Then, the smallest $\theta_{X Y}$ is defined as $\theta_{X Y \mathrm{c}}$. Note that $\theta_{X Y \mathrm{c}}$ can be expressed as a function of $d$. ${ }^{6}$ ) The effective cross-sectional area $D_{\text {eff }}$ is described by the diameter of the tether $D_{\mathrm{T}}$ and $\theta_{X Y \mathrm{c}}$. If the center of debris passes through $D_{\text {eff }}$, the damage will be enough to sever the hollow cylindrical tether. In case 3 , the damage to the hollow cylindrical tether caused by the debris collision increased as the $X Y$-plane impact angle $\theta_{X Y}$ increased. In other words, the damage is smallest at $\theta_{X Y}=0^{\circ}$. The critical $X Y$-plane impact angle $\theta_{X Y \mathrm{c}}$ describes $\theta_{X Y}$ at the moment when the residual ratio $R$ is equal to the critical one $R_{\mathrm{c}}$. If $R$ is already under $R_{\mathrm{c}}$ at $\theta_{X Y}=0^{\circ}$, then the hollow cylindrical tether would be severed by a debris collision at any $\theta_{X Y}$. Hence the effective cross-sectional area $D_{\text {eff }}$ is the diameter of the tether $D_{\mathrm{T}}$.

By summarizing the above three cases, the effective cross-sectional area $D_{\text {eff }}$ for both sides of the hollow cylindrical tether can be expressed as follows: ${ }^{6}$ )

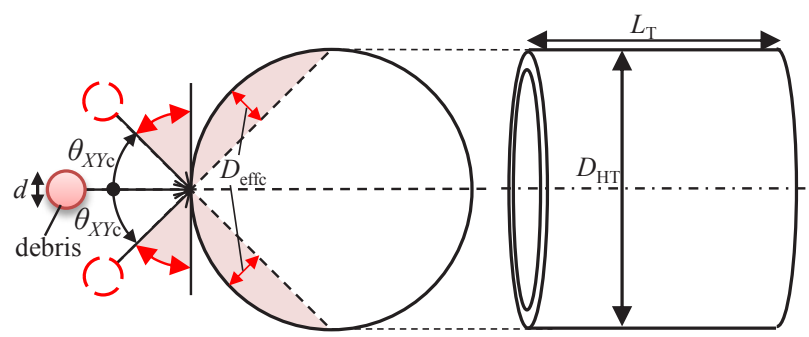

Fig. 12. Effective cross-sectional area of proposed tether (hollow cylindrical tether)

$$
D_{\mathrm{eff}}=\left\{\begin{array}{cl}
0 & \text { for } 90^{\circ}<\theta_{X Y \mathrm{c}} \\
D_{\mathrm{HT}}\left\{1-\sin \left(\theta_{X Y \mathrm{c}}\right)\right\} & \text { for } \quad 0^{\circ} \leq \theta_{X Y \mathrm{c}} \leq 90^{\circ} \\
D_{\mathrm{HT}} & \theta_{X Y \mathrm{c}} \text { has no solution }
\end{array} .\right.
$$

\section{Survival Probability of Hollow Cylindrical Tether}

\subsection{Probability of fatal impact on tether}

Survivability can be described in terms of three factors: the impact region of debris on the tether, the residence time in orbit, and the debris flux. The fatal impact rate $I_{\mathrm{f}}$ (i.e., the probability of an impact on the tether being fatal during a year) can be expressed as

$$
I_{\mathrm{f}}=-\int_{d_{\mathrm{m}}}^{d_{\infty}} L_{\mathrm{T}} D_{\mathrm{eff}}(d) \frac{\mathrm{d} F(d)}{\mathrm{d} d} \mathrm{~d} d,
$$

where $L_{\mathrm{T}} D_{\text {eff }}$ is the impact area. The debris flux model ORDEM $2000^{10)}$ developed by NASA was used to simulate the cumulative flux of the debris. The cumulative debris flux is the number of times that debris of a certain diameter $d$ or larger passes through a given area of $1 \mathrm{~m}^{2}$ in a year. The minimum debris diameter for the conventional tether is given by $f_{\mathrm{m}} D_{\mathrm{ST}}$, where $f_{\mathrm{m}}$ is the scale factor of the minimum debris diameter and is often set to about 0.33 or $1 / 3 .^{5,8)}$ The scale factor for the proposed tether is given by experimental results. $d_{\infty}$ is the maximum debris diameter provided by the ORDEM2000 debris flux model. The cumulative debris flux used in this study was: orbital inclination of $98^{\circ}$, year 2000 , and altitude of $800 \mathrm{~km}$. According to Anz-Meador ${ }^{11)}$, the survivability of the tether can be expressed as

$$
P_{0}=\exp \left[-I_{\mathrm{f}} \Delta t\right] \text {. }
$$

\subsection{Comparison of survival probability}

The survivability was calculated based on a scenario introduced by Khan and Sanmartin. ${ }^{8)}$ Figure 13 shows the

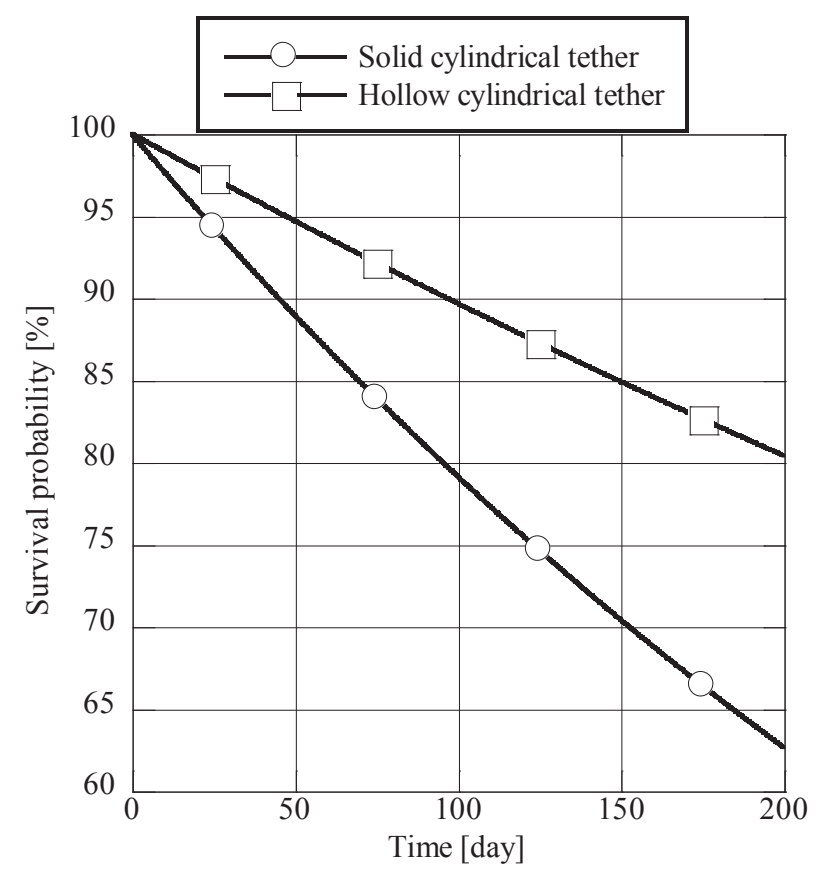

Fig. 13. Survival probabilities of two tethers at altitude of $800 \mathrm{~km}$. 
survivability values $P_{0}$ for the hollow and solid cylindrical tethers at an altitude of $800 \mathrm{~km}$. As shown in Ref. 6), $f_{\mathrm{c}}=0.33, f_{\mathrm{m}}=0.33$, and $d_{\infty}=1 \mathrm{~m}$ were employed. As shown in Ref. 8), $\alpha=0.253$ was employed. To compare the two types of tethers under identical conditions, the total mass of the solid cylindrical tether was equal to that of the hollow cylindrical tether. The diameters of the hollow and solid cylindrical tethers were set to $146 \mathrm{~mm}$ and $6.61 \mathrm{~mm}$, respectively. The lengths of both tethers were set to $10 \mathrm{~km}$. The mass was the same for both tethers. Here, the oblique collision with debris was not considered; $\theta_{Z X}=0^{\circ}$. The hollow cylindrical tether exhibited a higher survivability than the solid cylindrical tether. When 200 days have passed, the survival probability of the hollow cylindrical tether is over $15 \%$ higher than that of the solid cylindrical tether. This was attributed to the hollow structure that increases the tether diameter. The minimum debris diameter $d_{\mathrm{m}}$ was increased while the masses and lengths of the solid and hollow cylindrical tethers being the same. $d_{\mathrm{m}}$ of the solid cylindrical tether was $2.21 \mathrm{~mm}$, and $d_{\mathrm{m}}$ of the hollow cylindrical tether was $4.52 \mathrm{~mm}$. Thus, the protective performance of the hollow cylindrical tether against debris collision was greater. In addition, its usefulness in the debris removal missions is significantly improved compared to the conventional tether. In practice, the hypervelocity impact experiments validated that the hollow cylindrical tether is superior to the solid cylindrical tether, in terms of the debris collision only for this scale because there was no data for other scales. Therefore, if the hollow cylindrical tether is expected to be used for a debris removal mission, the influence of its diameter must be analyzed and considered to evaluate the survival probability accurately.

\section{Oblique Impact Experiments}

\subsection{Overview of oblique impact experiments}

In the previous section, the superiority of the hollow cylindrical tether was presented. However, the damage to a hollow cylindrical tether due to an oblique impact is considered to be larger than a horizontal impact. Figure 14 shows a comparison of the damaged areas for the horizontal and oblique impacts. The setup of the hypervelocity experiment of the hollow cylindrical tether and the projectile is shown in Fig. 4, section 3.2. The relationship between each impact angle of the projectile and the damage to the hollow cylindrical tether is described in the next subsection.

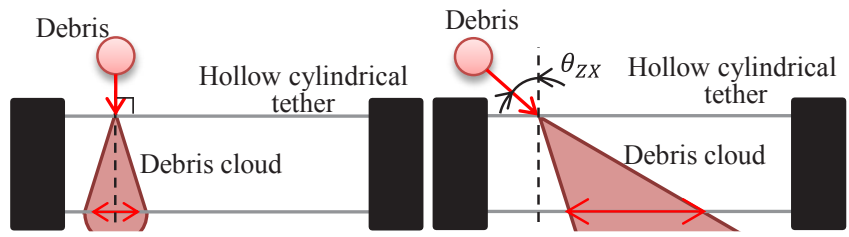

Fig. 14. Differences between debris-cloud-spreading angle and damaged area for horizontal and oblique impacts.

\subsection{Results of oblique impact experiments}

Figure 15 shows photographs of the hollow cylindrical tether after the hypervelocity oblique impact experiment. In Fig. 15(a), $\theta_{Z X}=0^{\circ}$ and $\theta_{X Y}=27.4^{\circ}$. In Fig. 15(b), $\theta_{Z X}=$ $30^{\circ}$ and $\theta_{X Y}=23.6^{\circ}$. In Fig. 15(c), $\theta_{Z X}=45^{\circ}$ and $\theta_{X Y}=$ $24.2^{\circ}$. The impact direction of the projectile was directed out of the sheet. As the $Z X$-plane angle $\theta_{Z X}$ increased, the 1 st and 2 nd destruction areas tended to increase. The volume of debris passing through the hollow cylindrical tether increased with an increase of the $Z X$-plane angle $\theta_{\mathrm{ZX}}$, hence the $1 \mathrm{st}$ destruction area caused by the debris impact increased. Accordingly, the amount of the debris cloud generated inside of the hollow cylindrical tether increased. The 2 nd destruction area caused by the debris-cloud impact increased, too. The shape of the 2 nd destruction varied with the variation of the $Z X$-plane angle $\theta_{Z X}$. When $\theta_{Z X}$ is $0^{\circ}, 30^{\circ}$, or $45^{\circ}$, then the shape was a circle, ellipse, or helix, respectively. This indicates the debris cloud moved along the inside surface of the hollow cylindrical tether with the tether being damaged. To prove this, the tracks of the debris cloud were focused upon. Figure 16 shows photographs taken by the high-speed camera when the conditions of the experiment were $\theta_{Z X}=$ $45^{\circ}$ and $\theta_{X Y}=24.2^{\circ}$. At the moment that debris impacted the tether $t=0 \mu \mathrm{s}$. At $t=16 \mu \mathrm{s}$, the debris cloud generated inside of the hollow cylindrical tether impacted on the inside surface of the hollow cylindrical tether. The debris cloud was observed to move along the inside surface of the hollow cylindrical tether as time passed, since it collided with the
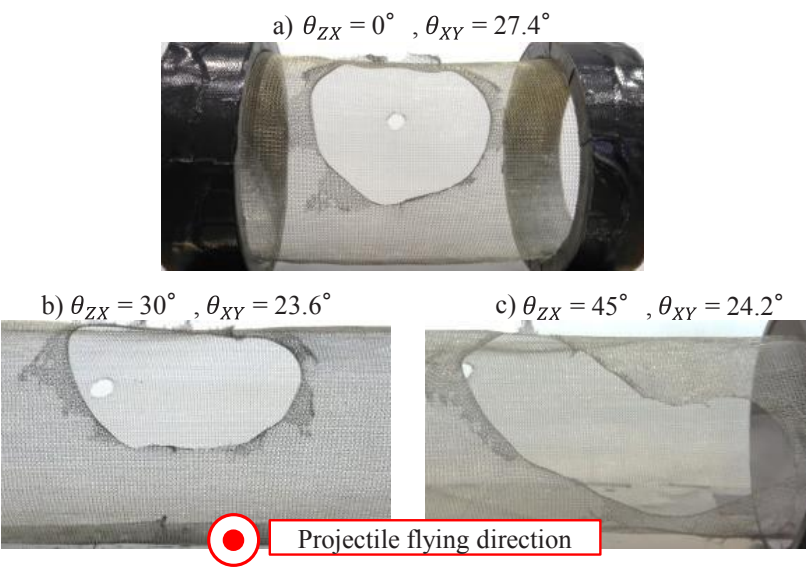

Fig. 15. Damage of tether after impact: a) $\theta_{Z X}=0^{\circ}, \theta_{X Y}=27.4^{\circ}$, b) $\theta_{Z X}=30^{\circ}, \theta_{X Y}=23.6^{\circ}$, and c) $\theta_{Z X}=45^{\circ}, \theta_{X Y}=24.2^{\circ}$.

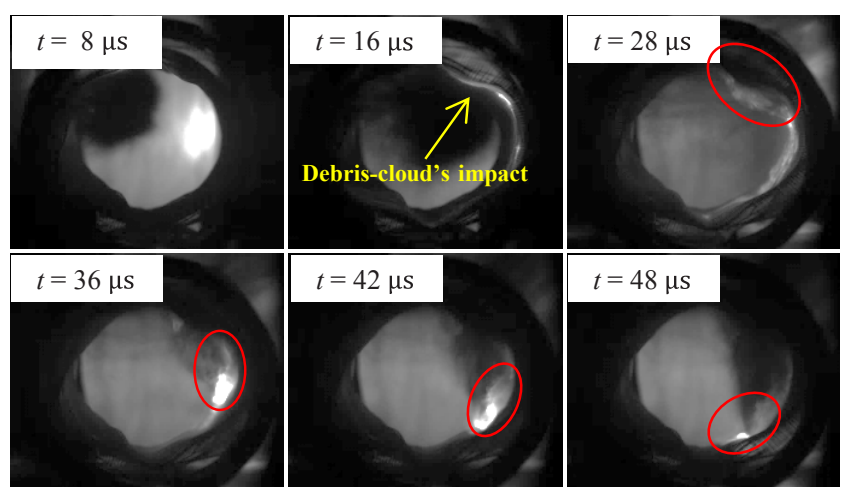

Fig. 16. Photographs of debris cloud ( $\left.\theta_{Z X}=45^{\circ}, \theta_{X Y}=24.2^{\circ}\right)$, where $t$ is past time after debris impact. 
inside surface of the tether. The circumferential velocity of the debris cloud was gradually decelerated after impacting the inside surface of the hollow cylindrical tether. This fact leads to an emphasis upon the longitudinal velocity. Hence the shape of the damage caused by the debris cloud extended to the longitudinal direction of the tether towards the jigs as shown in Fig. 15(c). The direction that the debris-cloud travelled depended on the impact angle, therefore the shape of the damage to the hollow cylindrical tether varied.

\subsection{Residual length by oblique impact}

Similar to subsection 3.4, the non-damaged circumferential ratio of the hollow cylindrical tether is defined as the residual ratio $R$. Figure 17 shows the relationship between the residual ratio $R$ and the $X Y$-plane impact angle $\theta_{X Y}$ at each $Z X$-plane impact angle $\theta_{Z X}$. At any $Z X$-plane impact angle $\theta_{Z X}$, as the $X Y$-plane impact angle $\theta_{X Y}$ increases, the residual ratio tends to decrease. In other words, as the distance between the 1st and 2nd destructions becomes smaller, as described previously, the damage increases because the debris cloud collides with a higher density. As the $Z X$-plane impact angle $\theta_{Z X}$ increases, although there are some variations, the residual ratio tends to decrease. The region impacted by the debris cloud became wider with an increase in the $Z X$-plane impact angle $\theta_{Z X}$.

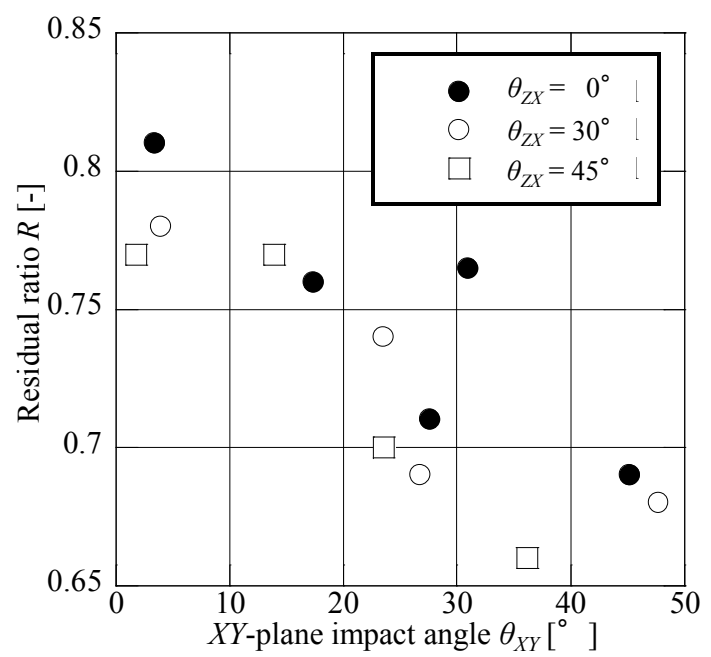

Fig. 17. Residual ratio $R$ vs $X Y$-plane impact angle $\theta_{X Y}$ for each $Z X$-plane impact angle $\theta_{Z X}$.

\section{Influence Evaluation of Debris Oblique Impact}

In section 6 , it was shown that the $Z X$-plane impact angle $\theta_{Z X}$ influences the damage to the hollow cylindrical tether. As the $Z X$-plane impact angle $\theta_{Z X}$ increased, the damage to the hollow cylindrical tether increased, in other words, the survival probability decreased.

To describe the increase of the damage to the hollow cylindrical tether against the oblique collision with debris, the effective cross-sectional area $D_{\text {eff }}$ is varied based on Fig. 18. For example, if the critical residual ratio $R_{\mathrm{c}}$ is set to 0.714 , the critical $X Y$-plane angle $\theta_{X Y_{\mathrm{c}}}$ is set to $28^{\circ}, 26^{\circ}$, or $22^{\circ}$ corresponding to $Z X$-plane impact angles $\theta_{Z X}, 0^{\circ}, 30^{\circ}$, or $45^{\circ}$, respectively. As described in Eq. (1), the effective cross-sectional area $D_{\text {eff }}$ depends on the critical $X Y$-plane angle $\theta_{X Y_{\mathrm{c}}}$. Hence, as the $Z X$-plane impact angle $\theta_{Z X}$ increased, the cross-sectional area $D_{\text {eff }}$ became larger. In addition, according to the oblique impact experimental results, the cross-sectional area $D_{\text {eff }}$ increased with increasing $Z X$-plane impact angle $\theta_{Z X}$.

The survival probabilities of each $Z X$-plane impact angle $\theta_{Z X}$ were calculated and are shown in Fig. 18. Then, the same scenario and coefficients of section 5 were adopted. A hollow cylindrical tether with a diameter of $146 \mathrm{~mm}$ and length of $10 \mathrm{~km}$ was used. The cumulative debris flux provided by ORDEM2000 used in this study was: orbital inclination of $98^{\circ}$, year 2000, and altitude of $800 \mathrm{~km}$. By calculating the survival probabilities under each condition, it was assumed that debris collided only at each angle (e.g. to calculate the survival probability of the $Z X$-plane impact angle $\theta_{Z X}=45^{\circ}$, debris collided only at $45^{\circ}$ ).

The survival probability decreased with an increase of $Z X$-plane impact angle $\theta_{Z X}$. This is because the minimum debris diameter $d_{\mathrm{m}}$ decreased with an increase of $Z X$-plane impact angle $\theta_{Z X}: d_{\mathrm{m}}$ was $4.52 \mathrm{~mm}, 4.42 \mathrm{~mm}$, or $4.22 \mathrm{~mm}$ when $\theta_{Z X}$ was set to $0^{\circ}, 30^{\circ}$, or $45^{\circ}$, respectively. This implies that the influence of the $Z X$-plane impact angle $\theta_{Z X}$ needs to be considered to assess the survival performance of a hollow cylindrical tether strictly.

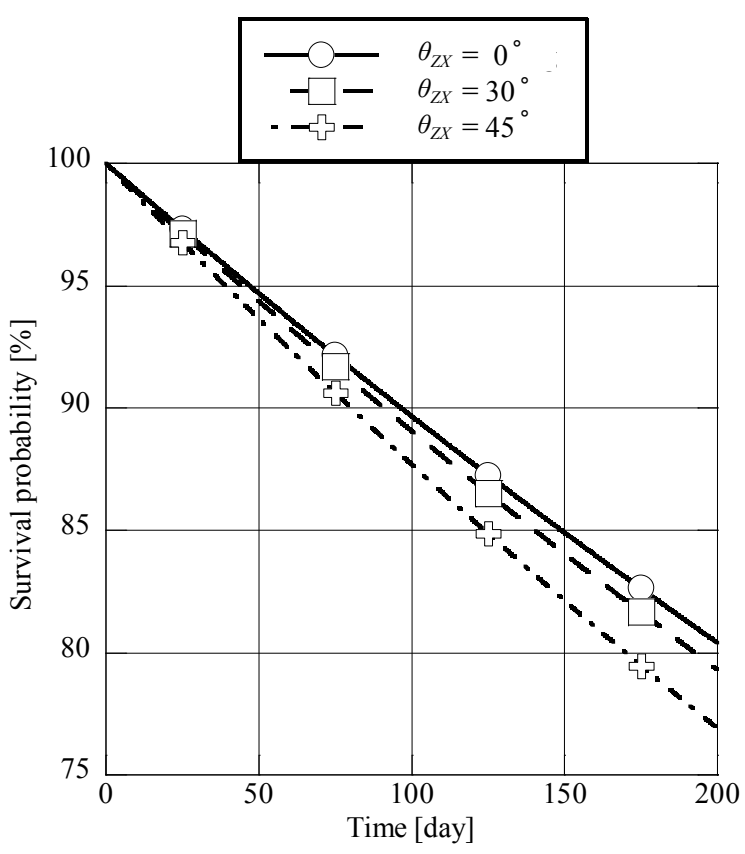

Fig. 18. Survival probabilities of hollow cylindrical tether at altitude of $800 \mathrm{~km}$.

\section{Conclusion}

A hollow cylindrical tether was investigated to improve protection against debris collision. To understand the relationship between the damage caused on the tether and the debris cloud generated inside of the tether, hypervelocity horizontal and oblique impact experiments were carried out. The experimental results revealed the influence of the impact 
angle of debris: the $X Y$ - and $Z X$-plane angles. The damaged area of the tether due to the debris-cloud impact was larger than the one due to the debris impact, and became larger when the impact angles increased. This is because the traveling distance of debris cloud became shorter. When the $Z X$-impact angle increased, especially, the debris cloud moved along the inside of the tether. This phenomenon can lead to larger damage to the tether due to a higher-density debris-cloud. To evaluate the damage caused on the tether, the residual ratio was used as an index. In addition, the survival probability was calculated and utilized for the comparison of solid and hollow cylindrical tethers. The results revealed that a hollow cylindrical tether was superior to a solid cylindrical tether, from the viewpoint of toughness against a small debris collision, because its critical debris diameter was larger. The circumferential damaged length depended on the $Z X$-plane impact angle as the spray range of debris cloud varied simply. A comparison of some of the $Z X$-plane impact angles on the hollow cylindrical tether proved that $Z X$-plane impact angle influences the damage to the hollow cylindrical tether. An increase of the $Z X$-plane impact angle led to a decrease in the success rate of the debris removal mission. Therefore, when the protective performance of a hollow cylindrical tether is evaluated, the influence of the $Z X$-plane impact angle should be considered.

\section{Acknowledgements}

The experiments in this study were conducted at Hypervelocity Impact Facility (former name: the Space Plasma Laboratory) of ISAS of JAXA. The experiments were also supported by and conducted in collaboration with ISAS/JAXA. Considerable assistance was received from the Collaborative Research Project of the Institute of Fluid Science, Tohoku University.

\section{References}

1) Hirayama, H., Kim, I., and Hanada, T.: Survivability of Tether throughout Deorbiting, Proceedings of International Symposium on Space Technology and Science, 2009-r-2-18, 2009.

2) Anselmo, L. and Pardini, C.: The Survivability of Space Tether Systems in Orbit Around the Earth, Acta Astronautica, 56(2005), pp. 391-396.

3) Anz-Meador, P. D.: Tether-Debris Interactions in Low Earth Orbit, Proceedings of the American Institute of Physics Conference, 552, 2001, pp. 525-531.

4) Pardini, C., Hanada, T., and Krisko, P.: Benefits and Risks of Using Electrodynamic Tethers to De-Orbit, Acta Astronautica, 64(2009), pp. 571-588.

5) Pardini, C., Hanada, T., Krisko, L., Anselmo, L., and Hirayama, H.: Are De-orbiting Missions Possible Using Electrodynamic Tethers? Task Reviews from the Space Debris Perspective, Acta Astronautica, 60(2007), pp. 916-929.

6) Makihara, K. and Matsumoto, N.: Survival Probability of Hollow Cylindrical Mesh Tether Under Space Debris Impact, J. Spacecraft and Rockets, 53(2016), pp. 520-527.

7) Ma, W., Zhang, W., and Pang, B.: Simulation of Characteristics of Debris Cloud Produced by Hypervelocity Impact, Advanced Materials Research, 940(2014), pp. 300-305.

8) Khan, S. B. and Sanmartin, J. R.: Survival Probability of Round and Tape Tethers Against Debris Impact, J. Spacecraft and Rockets, 50(2013), pp. 603-608.

9) Makihara, K. and Takahashi, R.: Survivability Evaluation of Electrodynamic Tethers Considering Dynamic Fracture in Space-Debris Impact, J. Spacecraft and Rockets, 53(2016), pp. 209-216.

10) Liou, J. C., Matney, M. J., Anz-Meador, P. D., Kessler, D., Jansen, M., and Theall, J. R.: The New NASA Orbital Debris Engineering Model ORDEM-2000, NASA/TP-2002-210780, 2002.

11) Anz-Meador, P. D.: Tether-Debris Interactions in Low Earth Orbit, Proceedings of the American Institute of Physics Conference, 552, American Inst. of Physics, Melville, NY, 2001, pp. 525-531. 